\title{
Numerical Simulation of Single Bubble Pool Boiling in Different Grav- ity Conditions
}

\author{
J. F. Zhao ${ }^{1, *}$, Z. D. Li $^{2}$, L. Zhang ${ }^{1}$ \\ ${ }^{1}$ Key Laboratory of Microgravity (National Microgravity Laboratory) / CAS; Institute of Mechanics, Chinese Academy \\ of Sciences (CAS), Beijing 100190, China \\ ${ }^{2}$ State Nuclear Power Technology Research \& Development Center, Beijing 100190, China
}

Email: jfzhao@imech.ac.cn

\begin{abstract}
In this paper, the growth processes of a single bubble on a fixed single site in different gravity are numerically investigated for saturated water at $0.1 \mathrm{MPa}$. The departure diameter decreases with the decrease of the wall superheat, while the growth period increases. Both the growth period and departure diameter decrease with the decrease of the contact angle, but increase with the decrease of gravity. The numerical results of single bubble boiling indicate that the heat transfer through the micro-wedge region has a very important contribution to the whole heat transfer in boiling. The area-averaged heat fluxes in different gravity are approximately proportional to the 1.5 th power of the wall superheat when the number density of active nucleation sites fixes, which is consistent with empirical correlation based on experiments in normal gravity.
\end{abstract}

Key words: nucleate pool boiling, bubble growth, departure diameter, level-set method

\section{INTRODUCTION}

Boiling heat transfer is one of the most efficient modes of heat transfer. Many correlations and semi-mechanistic models have also been developed for various modes of boiling. However, due to the complexity involved in modeling the boiling heat transfer phenomena, unrealistic assumptions are often made in developing various models. With the advances of recent years in the area of computational science and engineering, it is now possible to solve, completely, the conservation equations of mass, momentum, and energy for liquid and vapor phases simultaneously when an interface is continuously evolving at and near a heated surface. The solutions provide not only detailed physics of associated thermal and hydrodynamic processes, but also the shape of the evolving interface.

Up to now, numerical simulations for directly analyzing the bubble dynamics and heat transfer in single bubble nucleate boiling have been performed by several investigators [1-8], most of them are focus on the normal gravity level. The aim of the present study is to simulate the single bubble boiling phenomena under variable gravity condition, wall superheat, contact angle etc. A complete numerical simulation of the growth and departure of single bubble on a horizontal heated surface during pool boiling under variable gravity conditions is proposed here. The effects of reduced gravity condition, wall superheat and contact angle on the bubble diameter, interfacial structure, bubble departure time, as well as heat fluxes are to be studied.

\section{MATHEMATICAL FORMULATION AND NUMERICAL METHOD}

Heat transfer through the three-phase contact line area has very large contribution to the overall heat transfer of boiling, so, in the simulation, the region of interest is divided into micro and macro regions [8]. The micro region is the ultra thin liquid film that forms between the solid surface and the evolving liquid-vapor interface. The macro region is the region occupied by vapor and liquid, except the micro region. For the macro region, complete conservation equations 
of mass, momentum, and energy are solved for both phases. Interface position is captured through a level set function.

Lubrication theory is employed to set up the micro region model, and it has been used and validated by a number of investigators in the literature, such as Wayner [9], Lay and Dhir [10]. Son et al. [3] pointed out that the interface in the micro region has a nearly constant slope except near the non-evaporating region where the slope decreases to zero rapidly. Thus Son [11] assumed the interface slope in the micro region is constant and then he deduced a simplified 2-D planar model for the micro region. According to the same idea, a 2-D axisymmetric model for the micro region is here deduced as follows,

$$
\begin{aligned}
& \dot{V}_{\text {mic }}=\frac{2 \pi k_{1}\left(T_{\mathrm{w}}-T_{\mathrm{sat}}\right)}{\rho_{\mathrm{v}} h_{\mathrm{fg}} \Delta V_{\mathrm{mic}}}\left[\left(R_{1}-\frac{h}{2 \tan \phi}\right) \frac{1}{\tan \phi} \ln \left(\frac{h}{2} \frac{h_{\mathrm{ev}}}{k_{1}}+1\right)+\frac{h}{2 \tan ^{2} \phi}-\frac{k_{1}}{h_{\mathrm{ev}}} \frac{1}{\tan ^{2} \phi} \ln \left(\frac{h}{2} \frac{h_{\mathrm{ev}}}{k_{\mathrm{l}}}+1\right)\right] \\
& q_{\text {mic }}=\frac{2 k_{\mathrm{l}}\left(T_{\mathrm{w}}-T_{\mathrm{sat}}\right)}{L^{2}}\left[\left(R_{1}-\frac{h}{2 \tan \phi}\right) \frac{1}{\tan \phi} \ln \left(\frac{h}{2} \frac{h_{\mathrm{ev}}}{k_{\mathrm{l}}}+1\right)+\frac{h}{2 \tan ^{2} \phi}-\frac{k_{1}}{h_{\mathrm{ev}}} \frac{1}{\tan ^{2} \phi} \ln \left(\frac{h}{2} \frac{h_{\mathrm{ev}}}{k_{\mathrm{l}}}+1\right)\right]
\end{aligned}
$$

where $q_{\text {mic }}$ is the heat production of the micro region which averaged by the whole heat surface.

For the macro region, the level set formulation is used. The interface separating the two phases is captured by a level set function, $\phi$. The discontinuous pressure drop across vapor and liquid caused by surface tension force is smoothed into a numerically continuous function using the continuum interface model. The computational domain was chosen to be $(1 L, 3 L)$, whear $L=\sqrt{\sigma / g\left(\rho_{1}-\rho_{2}\right)}$. A grid number of $100 \times 300$ is used. The computational precision is verified to be guaranteed, and also that bubble growth is not affected by the computational boundary. Bubble growth and heat transfer during pool boiling of saturated water with a single bubble are computed in the present study. A constant contact angle of $38^{\circ}$ is used in the present study. The projection method [12] is used to solve the governing equations. To eliminate spurious currents caused by the effect of surface tension, we use the PBM method [13]. The detailed equations, initial and boundary conditions, as well as the solving algorithm, can be found in Ref.[14].

\section{RESULTS AND DISCUSSION}

Figure 1 shows two different bubble growth curves corresponding to initial radius of $0.1 \mathrm{~L}$ and $0.05 \mathrm{~L}$, respectively. The results indicate that the initial bubble radius has obvious effects only on a much small part of the early growth period, which reflects the great departure of the still initial condition adopted in the study from the real condition of the forming of initial bubble. During the other part of the growth period, no deffience can be found between these two cases. The bubble departure diameter and the growing time before its departure have the same results. Thus, it can be concluded that the influence of the initial bubble radius is negligible if an adequately small value is provided. In the following calculations, $0.1 \mathrm{~L}$ is chosen for the case of $1 \mathrm{~g}$ and $0.1 \mathrm{~g}$, while $0.05 \mathrm{~L}$ for the case of $0.01 \mathrm{~g}$.

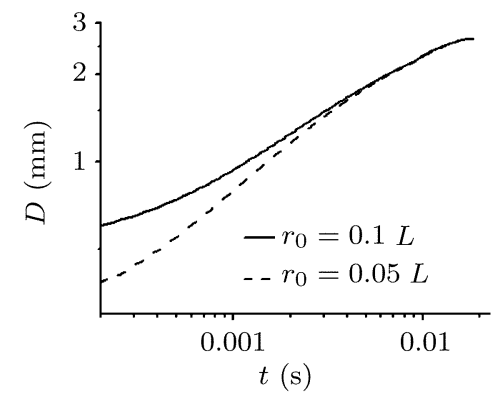

Figure 1: Bubble growth curves for different initial bubble radius

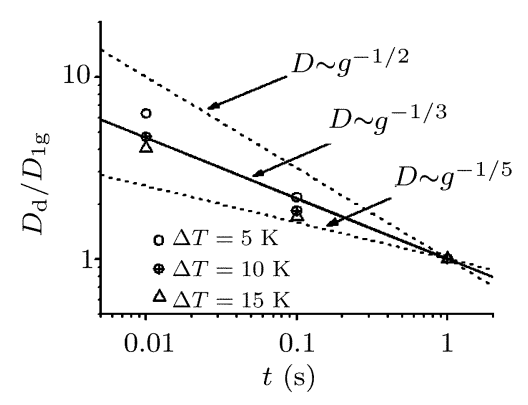

Figure 2: Bubble departure diameter vs. gravity level

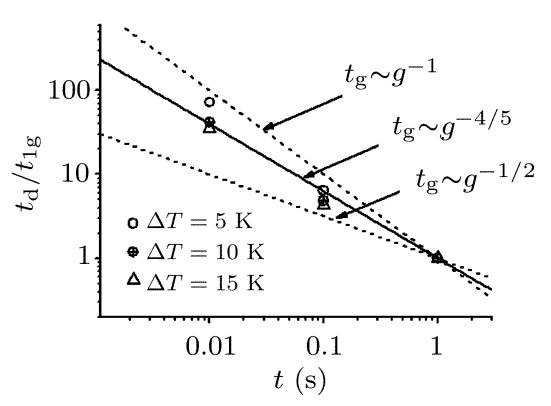

Figure 3: Bubble growth time vs. gravity level

The magnitude of gravitational acceleration is an important variable that can affect the bubble growth and departure process. The numerical simulations are also used to study the effect on bubble dynamics of the magnitude of gravitational acceleration normal to the heater surface. The present results, shown in Figure 2, are consistent with Siegel \& Keshock [15], who found in microgravity experiments that the bubble departure diameter is approximately proportional to the $1 / 3$ power of gravity. Figure 3 shows that the bubble growth time is approximately proportional to the $-4 / 5$ power of gravity. 
The total heat flux from the wall and the contributions from the micro region in different gravity are plotted in Figure 4. The results indicate that heat fluxes have the same trend. During the early period of bubble growth, because of the increasing of micro region area, heat flux increases with time, while heat flux decreases with time during the latter period of bubble growth due to the decreasing of micro region area caused by the lifted bubble. After the bubble detaches, heat transfer returns to natural convection. The results indicate that the heat transfer from the micro region has a very important contribution to the whole heat transfer in boiling, and must be considered in the simulation and modeling.
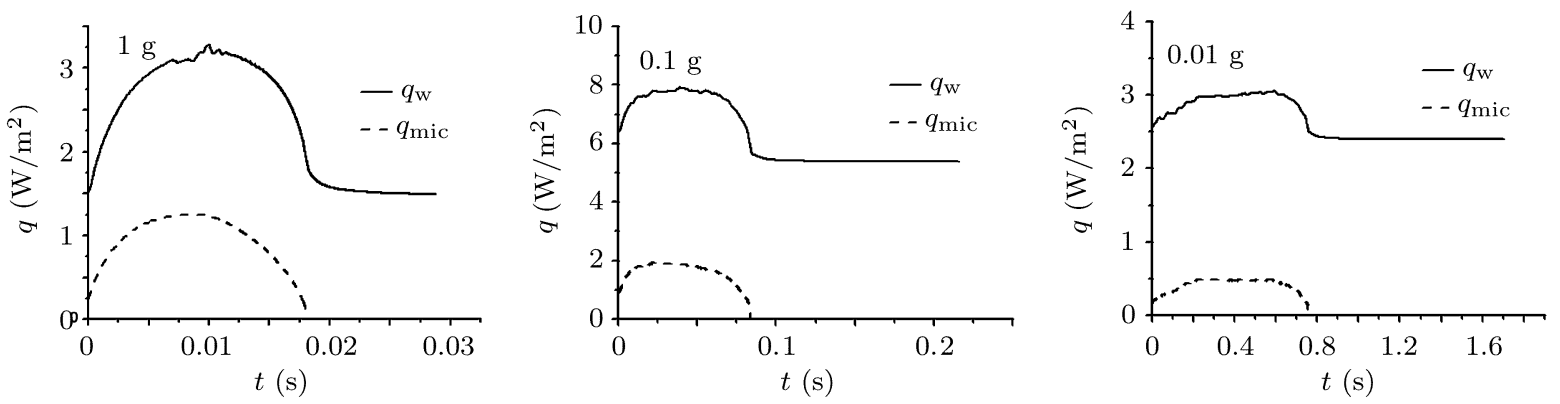

Figure 4: Averaged wall heat flux and those contributing from the micro region at $\Delta T=10 \mathrm{~K}$

To cumpute the averaged heat flux during a period of bubble life, the correlations of Zuber [16] and of Di Marco and Grassi [17], namely $f D_{\mathrm{d}}=0.59\left[\sigma g\left(\rho_{\mathrm{l}}-\rho_{\mathrm{v}}\right) / \rho_{1}^{2}\right]^{1 / 4}$ and $f_{\mathrm{m}} / f=\left(g_{\mathrm{m}} / g\right)^{0.75}$, are used for the departure frequency of growing bubble in normal gravity and for the case in partial gravity conditions, respectively. Thus, the averaged heat flux during a period of bubble life can be computed as $\underline{q}=Q / \pi L^{2}=\int_{0}^{1 / f_{\mathrm{m}}} q \mathrm{~d} t$.

Figure 5 shows the comparison between the present results and the common-used Rohsenow correlation [18] in normal gravity. It is found that the present results are consistent with the predictions of Rohsenow's correlation with the empirical parameter $C_{s}=0.02$. However, further analysis shows that Rohsenow's correlation indicatesa 3rd-power dependence on heat flux on the wall superheat, while the present numerical results exhibits a 1.5th-power dependence. The reason lies in the following fact. In the simulation, the single bubble model is used and then the effects of the number density of active nucleate sites cannot be considered. Following Zuber [19], $q=C \Delta T^{n} N_{a}^{m}$, where $C, m$, and $n$ are empirical constants, while $N_{a}$ is the number density of active nucleate sites. As suggested by Zuber [19], $n=3 / 2$, $m=1 / 4$ for the case of low heat flux, while $n=5 / 3, m=1 / 3$ for the case of high heat flux. Obviously, the single bubble model ought to be corresponding to the case of low heat flux, and the numerical results are consistent perfectly with Zuber's suggestion. Furthermore, this trend is preserved in reduced gravity conditions, at least up to 0.01g (Figure $6)$.

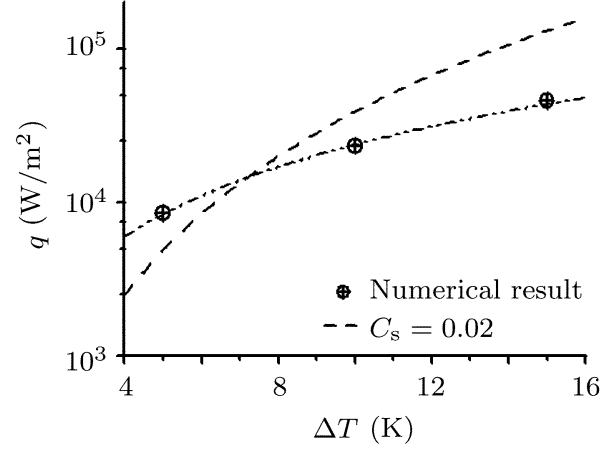

Figure 5: Comparison between the present result and the commonused Rohsenow correlation in normal gravity

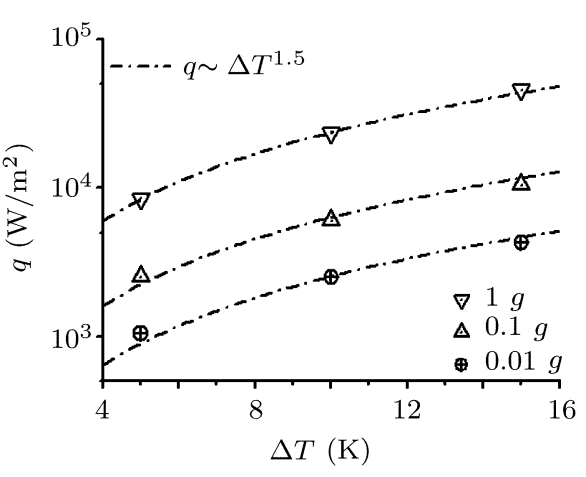

Figure 6: Heat flux in different gravity

\section{CONCLUSIONS}

Bubble growth and heat transfer during pool boiling of saturated water with a single bubble are computed in the present study. The numerical results indicate that the heat transfer through the micro-wedge region has a very important contribution to the whole heat transfer in boiling. The departure diameter decreases as wall superheat decreases, while the growing time increases. Both of the growing time and departure diameter increase as gravity decreases. The 
numerical results indicate that heat fluxes in different gravity conditions are proportional to the 1.5 th power of the wall superheat when the number density of active nucleation sites fixed, which are consistent perfectly with Zuber's suggestion.

\section{Acknowledgements}

The support of the National Natural Science Foundation of China (10972225) is gratefully acknowledged.

\section{REFERENCES}

1. Lee RC, Nydahl JE. Numerical calculation of bubble growth in nucleate boiling from inception through departure. J. Heat Transfer, 1989; 111: 474-479

2. Welch SWJ. Direct simulation of vapor bubble growth. Int. J. Heat Mass Transfer, 1998; 41: 1655-1666

3. Son G, Dhir VK, Ramanujapu N. Dynamics and heat transfer associated with a single bubble during nucleate boiling on a horizontal surface. J. Heat Transfer, 1999; 121: 623-632

4. Son G, Ramanujapu N, Dhir VK. Numerical simulation of bubble merger process on a single nucleation site during pool nucleate boiling. J. Heat Transfer, 2002; 124: 51-62

5. Mukherjee A, Dhir VK. Study of lateral merger of vapor bubbles during nucleate pool boiling. J. Heat Transfer, 2004; 126: 1023-1039

6. Abarajith HS, Dhir VK, Son G. Numerical simulation of the dynamics of multiple bubble merger during pool boiling under reduced gravity conditions. Proc. 7th Japan-U.S. Seminar on Two-Phase Flow Dynamics, Moriyama, Japan, 2004

7. Fuchs T, Kern J, Stephan P. A transient nucleate boiling model including microscale effects and wall heat transfer. J. Heat Transfer, 2006; 128: 1257-1265

8. Stephan P, Hammer J. A new model for nucleate boiling heat transfer. Wärme- und Stoffübertragung, 1994; 30: $119-125$

9. Wayner PC. Evaporation and stress in the contact line region. Proc. Eng. Fund. Conf. on Pool \& Flow Boiling, ASME, New York, 1992, 251-256

10. Lay J H, Dhir VK. Numerical calculation of bubble growth in nucleate boiling of saturated ILiquids. J. Heat Transfer, 1995; 117: 394-401

11. Son G. Numerical study on a sliding bubble during nucleate boiling. KSME Int. J., 2001; 15(7): 931-940

12. Son G, Dhir VK. Numerical simulation of film boiling near critical pressures with a level set method. $J$ Heat Transfer, 1998; 120: 183-192

13. Wang ZY. Numerical Study on Capillarity-Dominant Free Surface and Interfacial Flows. [Ph.D. Thesis], The University of Texas at Arlington, USA, 2006.

14. Li ZD. Numerical Simulation on Thermo-dynamical Behaviors of Bubbles in Different Gravity Conditions. [Ph.D. thesis], Institute of Mechanics, Chinese Academy of Sciences, Beijing, China, 2010

15. Siegel R, Keshock EG. Effects of reduced gravity on nucleate boiling bubble dynamics in saturated water. $J$. AIChE, 1964; 10(4): 509-517

16. Zuber N. Hydrodynamic Aspect of Boiling Heat Transfer. [Ph. D. Thesis], University of California, Los Angeles. 1959

17. Di Marco P, Grassi W. Pool boiling in microgravity: assessed results and open issues. Proc. 3rd European Thermal Sc. Conf., 2000

18. Mikic BB, Rohsenow WM. A new correlation of pool boiling data, including the effect of heating surface characteristics. J. Heat Transfer, 1969; 9: 245-250

19. Zuber N. Nucleate boiling - the region of isolated bubbles and the similarity with natural convection. Int. J. Heat Mass Transfer, 1963; 6(1): 53-60 